\title{
Ultra-high four wave mixing efficiency in slot waveguides with silicon nanocrystals
}

A. Trita, C. Lacava, P. Minzioni, J.-P. Colonna, P. Gautier, J.-M. Fedeli, and I. Cristiani

Citation: Appl. Phys. Lett. 99, 191105 (2011); doi: 10.1063/1.3659694

View online: http://dx.doi.org/10.1063/1.3659694

View Table of Contents: http://aip.scitation.org/toc/apl/99/19

Published by the American Institute of Physics

\section{Articles you may be interested in}

Third-order nonlinearities in silicon at telecom wavelengths

Applied Physics Letters 82, 2954 (2003); 10.1063/1.1571665

Low-power four-wave mixing in porous silicon microring resonators

Applied Physics Letters 109, 021106 (2016); 10.1063/1.4958698

Optical dispersion, two-photon absorption and self-phase modulation in silicon waveguides at $1.5 \mu \mathrm{m}$ wavelength

Applied Physics Letters 80, 416 (2002); 10.1063/1.1435801

Enhanced four-wave mixing in graphene-silicon slow-light photonic crystal waveguides

Applied Physics Letters 105, 091111 (2014); 10.1063/1.4894830

Stimulated and spontaneous four-wave mixing in silicon-on-insulator coupled photonic wire nano-cavities Applied Physics Letters 103, 031117 (2013); 10.1063/1.4812640

Two-photon absorption and Kerr coefficients of silicon for $850-2200 \mathrm{~nm}$

Applied Physics Letters 90, 191104 (2007); 10.1063/1.2737359

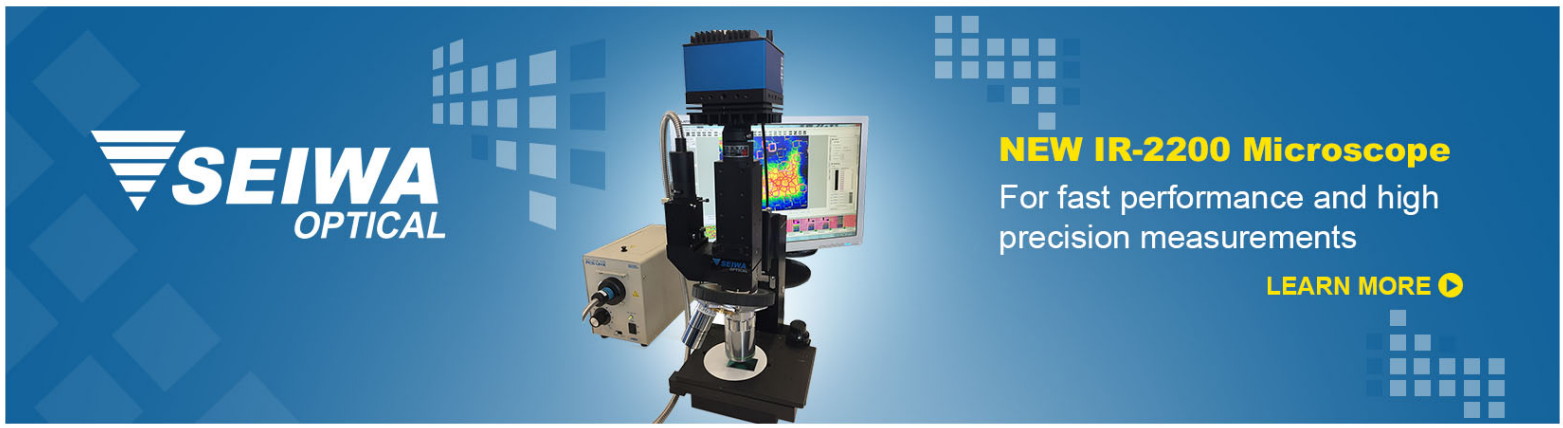




\title{
Ultra-high four wave mixing efficiency in slot waveguides with silicon nanocrystals
}

\author{
A. Trita, ${ }^{1, a)}$ C. Lacava,${ }^{1}$ P. Minzioni, ${ }^{1}$ J.-P. Colonna,${ }^{2}$ P. Gautier,${ }^{2}$ J.-M. Fedeli, ${ }^{2}$ \\ and I. Cristiani ${ }^{1, b)}$ \\ ${ }^{1}$ Dipartimento di Elettronica, Università di Pavia, Pavia 27100, Italy \\ ${ }^{2}$ CEA LETI, Minatec Campus, Grenoble 38054, France
}

(Received 27 September 2011; accepted 17 October 2011; published online 9 November 2011)

\begin{abstract}
We report on wavelength conversion through four-wave-mixing in silicon slot-waveguides with embedded silicon nanocrystals. The combination of strong optical confinement and Si:nc nonlinearity provides a huge waveguide nonlinear coefficient $\gamma=1100 \mathrm{~W}^{-1} \mathrm{~m}^{-1}$. Moreover, the improvement in the fabrication procedure allowed a loss reduction with respect to previous reported structures, enabling the achievement of an extreme value for the conversion efficiency which represents the best result ever reported in the scientific literature. (C) 2011 American Institute of Physics. [doi:10.1063/1.3659694]
\end{abstract}

The development of highly nonlinear integrated devices is a key step towards the achievement of ultra-fast and lowpower-consumption optical signal processing in silicon ( $\mathrm{Si}$ ) chips. In order to attain such a goal, several constraints have to be fulfilled: the demand for miniaturization calls for structures with very-high refractive index contrast and extreme nonlinear efficiency. In addition, the required fabrication procedure should be completely compatible with complementary metal-oxide semiconductor (CMOS) processes, so as to allow a complete integration of the nonlinear optical elements with additional optical and electronic components.

Silicon-based slot-waveguides provide the unique capability to confine the optical field in a nano-scale region filled with a low-refractive-index material. ${ }^{1}$ The strong confinement achieved by using these structures, in combination with the insertion of a highly nonlinear material inside the slot, can in principle give rise to optical waveguides featuring very large values of the nonlinear coefficient $\gamma$. Following this research line, high nonlinearities has been also observed in a nano/slot waveguides exploiting different materials approaches, ${ }^{2-5}$ and particularly interesting results have been recently reported on slot waveguides having a nonlinear polymer embedded in the slot region. ${ }^{6}$

A different approach to the realization of highly nonlinear slot waveguides is based on the exploitation of a thin $(<100 \mathrm{~nm})$ layer of silica incorporating silicon nanocrystals (Si:nc) in the slot. Such a material, besides being completely compatible with a CMOS fabrication process, in principle guarantees a nonlinear refractive index parameter $n_{2}$ of the order of $10^{-17} \mathrm{~m}^{2} \mathrm{~W}^{-1}$, only slightly smaller than that exhibited by some nonlinear polymers and still one order of magnitude larger than that of silicon. According to Ref. 7, the presence of Si:nc could be particularly beneficial for the enhancement of the nonlinear properties thanks to the strong quantum-confinement effect induced by the very small size of silicon nanocrystals. A recent work has demonstrated the

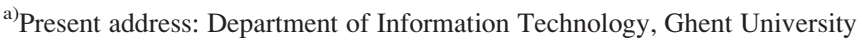
- IMEC, 9000 Gent, Belgium.

b) Author to whom correspondence should be addressed. Electronic mail: ilaria.cristiani@unipv.it.
}

possibility to realize all optical switching by means of ring resonators based on Si:nc slot waveguides. ${ }^{8}$ Particularly attractive is the possibility of performing wavelength conversion in the optical communication band. Indeed, a preliminary four-wave-mixing (FWM) experiment has shown the generation of a wavelength-converted signal in a Si:nc slot waveguide, with a conversion efficiency of $-47 \mathrm{~dB}{ }^{9}$

In this work we present the results of a FWM experiment performed by using Si:nc slot waveguides fabricated with an improved process that considerably reduces the linear losses. A key role is played by the fine control of the hydrogen content in the top amorphous-Si layer that allows saturating the dangling bonds responsible for mid-bandgap absorption in the infrared wavelength range. ${ }^{10,11}$ The nonlinear interactions were studied in both Transversal Magnetic-Field (TM) and Transversal Electric-Field (TE) case. In the TM case (slot confined mode), the measured conversion efficiency exceeds by about $18 \mathrm{~dB}$ the one obtained in the previous experiment. ${ }^{9}$ The value of the nonlinear waveguide coefficient derived from the experimental results is $\gamma=1100 \mathrm{~W}^{-1} \mathrm{~m}^{-1}$ that is the highest ever measured for uniform waveguides.

The sample under investigation consists of a 5-mm-long Si:nc slot waveguide, with a $100-\mathrm{nm}$ thick slot section, deposited by plasma-enhanced chemical vapor deposition (PECVD), embedded between two silicon slabs with a thickness of $220 \mathrm{~nm}$ (bottom slab) and $240 \mathrm{~nm}$ (top slab). An image of the structure, recorded by a scanning-electron microscope, is reported in the inset of Fig. 1. The Si:nc (with an average nanocrystals size of $4 \mathrm{~nm}$ ) layer was obtained by annealing at $1000{ }^{\circ} \mathrm{C}$ in $\mathrm{N}_{2}$ ambient a Si-rich oxide $(16 \%$ excess silicon) for $210 \mathrm{~s}$. The refractive index of the layer measured with a Metricon MLines equipment is $\mathrm{n} \approx 1.6$.

The top slab of the waveguide is constituted by a layer of hydrogenated amorphous silicon, whose refractive index was matched to that of crystalline silicon by a fine tuning of the deposition conditions. The hydrogen content was optimized to obtain minimal propagation losses. Sheet losses below $1 \mathrm{~dB} / \mathrm{cm}$ were measured by M-lines technique both in the as-deposited layer and after annealing at temperature below $400^{\circ} \mathrm{C}$ for $1 \mathrm{~h}$. Higher temperatures or longer annealing times were found to increase losses, as hydrogen degases 


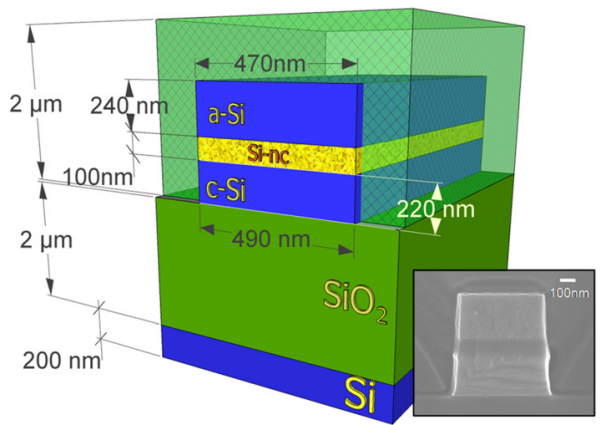

FIG. 1. (Color online) Scheme of the Si-nc slot waveguide. c-Si and a-Si indicate, respectively, crystalline silicon and amorphous silicon. The inset shows a SEM image of the overall structure.

and the crystallization process starts. The final slot waveguide has a $1 \mathrm{~mm}$ long input and output taper section to improve the coupling efficiency, yielding to an overall length of $7 \mathrm{~mm}$.

The experimental set-up used to test FWM conversion is shown in Fig. 2. Two external-cavity CW-lasers are separately amplified, polarization-controlled, and then coupled by $90: 10$ fiber coupler. The output beam is collimated by a gradedindex (GRIN) lens and then polarized by a Glan-Thompson polarizer, so that the polarization of the radiation launched into the waveguide can be properly controlled. The beam is then input to the waveguide via a $40 \times$ microscope infraredobjective (IR-objective). In order to monitor the power of both beams a $10 \%$ beam-splitter is positioned after the GlanThompson polarizer. The radiation output from the waveguide is collected by a $40 \times$ IR-objective and sent to a GRIN-lens fiber collimator, which is connected to an optical spectrum analyzer (OSA). In order to properly evaluate the nonlinear optical properties of the waveguide, we first accurately characterized coupling and propagation losses by measuring the transmission of a dedicated test structure composed of a $1 \times 2$ Multi-Mode Interference (MMI) $3 \mathrm{~dB}$ splitter followed by two Si:nc slot waveguides with different lengths. The measured values of the coupling losses, assumed identical for input and output, are $15.1 \mathrm{~dB}$ for the TM polarization and $14.5 \mathrm{~dB}$ for the TE polarization. Concerning the propagation losses the measured values are 3.9 and $6.9 \mathrm{~dB} / \mathrm{cm}$ for the quasi-TM and quasi-TE mode, respectively. Starting from these results, we calculated the effective length $\left(L_{e f f}\right)$ over which the nonlinear interaction is efficient. ${ }^{12}$ For the quasiTM mode, we found $L_{\text {eff }}=4 \mathrm{~mm}$, which is $80 \%$ of the actual waveguide length, so that a considerable portion of the waveguide is contributing to the FWM process. This result, due to the reduced losses of the hydrogenated amorphous silicon slab, represents an important break-through. In previous works, ${ }^{8,9}$ the estimated loss was of the order of $10 \mathrm{~dB} / \mathrm{cm}$ or

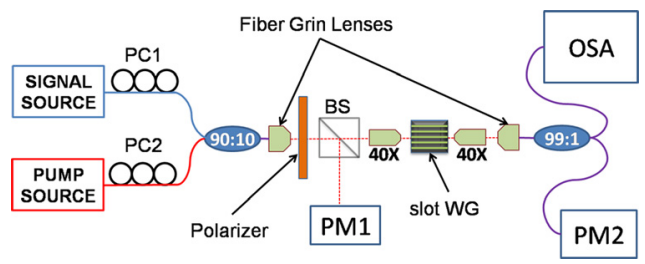

FIG. 2. (Color online) Experimental setup. PC1 and PC2 are two fiber polarization controllers; PM1 and PM2 are two power-meters and OSA is the optical spectrum analyzer. higher, leading to a $L_{\text {eff }}$ for the mode confined in the slot equal to about half of the waveguide length.

FWM measurements were first done by setting the pump and the signal wavelengths at, respectively, $\lambda_{p}=1550.10 \mathrm{~nm}$ and $\lambda_{s}=1550.30 \mathrm{~nm}$ and by recording the OSA traces of the radiation spectrum at the output of the $7-\mathrm{mm}$ chip for both input polarizations. The use of a so small detuning between pump and signal allows keeping as small as possible the phase-mismatch for the nonlinear process, hence maximizing the FWM efficiency for a given pump power.

The largest conversion efficiency was obtained when launching the pump and signal on the quasi-TM mode, yielding an overall FWM efficiency $\eta=-28.9 \mathrm{~dB}$ (defined as the ratio between idler and signal power, both measured at the waveguide output) when a pump power of $7 \mathrm{dBm}$ is launched at the waveguide section input (see Fig. 3). In similar conditions, the best efficiency recorded by polarizing both beams on the quasi-TE was about $-43 \mathrm{~dB}$.

By means of numerical simulations, carried out with the commercial tool Fimmwave by Photon Design, we evaluated the field distribution for the two orthogonal states of polarization of the optical beam. The quasi-TE mode is mainly localized in the silicon slabs and it has an effective area $A_{\text {eff }, T E}=0.23 \mu \mathrm{m}^{2}$. On the other hand, the quasi-TM mode is strongly confined in the Si:nc layer with an effective area that is about half that of the quasi-TE mode (effective area $A_{\text {eff,TM }}=0.13 \mu \mathrm{m}^{2}$ ).

To compare the obtained results with other values reported in the scientific literature, it is helpful to define a "normalized conversion efficiency" $\left(\eta_{\text {norm }}\right)$ which is independent of the used pump power $\left(P_{\text {pump }}\right)$ and of the device length $(L)$

$$
\eta_{\text {norm }}=\frac{100 \times P_{\text {idler }} / P_{\text {signal }}}{P_{\text {pump }}^{2} L^{2}} .
$$

In our case, we find $\eta_{\text {norm }}=112.15 \% \mathrm{~W}^{-2} \mathrm{~mm}^{-2}$, which seems to be the highest value ever experimentally measured. For sake of comparison, we recall that a value of $23.78 \% \mathrm{~W}^{-2} \mathrm{~mm}^{-2}$ was reported for GaInP photonic crystal waveguides ${ }^{13}$

From the obtained data, we were able to assess the parameter $\gamma$ for the quasi-TE and quasi-TM modes $\left(\gamma_{\mathrm{TE}}\right.$ and $\gamma_{\mathrm{TM}}$ in the following) by numerically solving the nonlinear equations describing the FWM phenomenon including the losses. ${ }^{12}$ In the numerical simulations, we neglected the phase mismatch terms, assuming that the wavelength detuning between

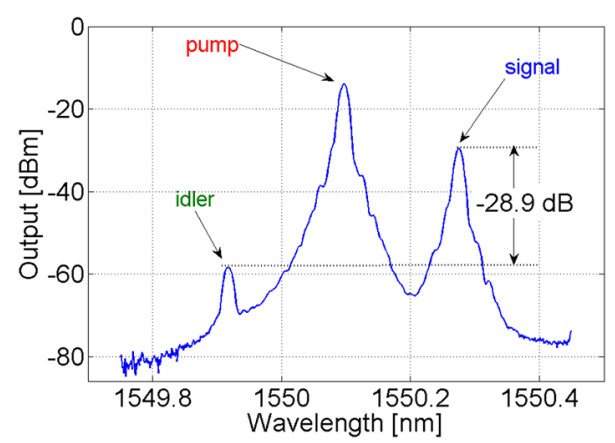

FIG. 3. (Color online) Optical spectrum acquired by the OSA. The ratio between the idler and signal power at the waveguide output is $-28.9 \mathrm{~dB}$, as shown in the picture. 
the pump and the signal was sufficiently small to guarantee the phase-matching condition. The value obtained for $\gamma_{\mathrm{TM}}$ is $1100 \mathrm{~W}^{-1} \mathrm{~m}^{-1}$, whereas $\gamma_{\mathrm{TE}}$ is one order of magnitude lower. Starting from these results, we could also roughly evaluate the Si:nc nonlinear coefficient $\mathrm{n}_{2}$ by using the relation

$$
n_{2}=\gamma A_{e f f} \frac{c}{\omega} \text {. }
$$

By inserting the value for the effective area derived from the numerical simulation, we find $n_{2 T M}=3.7 \times 10^{-17} \mathrm{~m}^{2} \mathrm{~W}^{-1}$ which is very close to previously reported results. ${ }^{14}$ It is also interesting to note that by applying the same procedure to $\gamma_{T E}$, we obtain $n_{2 T E}=6.2 \times 10^{-18} \mathrm{~m}^{2} \mathrm{~W}^{-1}$, which is very similar to the $n_{2}$ value of silicon, as expected, since the TE mode is mainly confined in the two Si-slabs.

In order to characterize the bandwidth of FWM process for the TM-polarization, we set the pump wavelength at $\lambda_{\mathrm{P}}=1532 \mathrm{~nm}$ and we progressively shifted the signal up to a distance of about $42 \mathrm{~nm}$ from $\lambda_{\mathrm{P}}$ (the wavelength shift was limited by the band of the optical amplifiers). The amplitude of the converted signal, normalized to the efficiency obtained at 0.2-nm detuning, is shown in Fig. 4. The experimental results show that for a $5-\mathrm{mm}$ long waveguide, the $-3 \mathrm{~dB}$ FWM-bandwidth is $\approx 10 \mathrm{~nm}$.

The conversion bandwidth is essentially controlled by the group velocity dispersion $\left(\beta_{2}\right)$ parameters. Silicon-based waveguides usually operate in normal dispersion with a very high $\beta_{2}$ value, but the waveguide dispersion contribution can be finely tuned in order to mitigate silicon $\beta_{2}{ }^{15}$ Numerical simulations reported in recent works indicate that the fabrication parameters leading to low and flat group-velocity-dispersion in Si:nc slot waveguides are less critical than in silicon wires. In addition, such parameters still allow obtaining an optimal nonlinear performance. ${ }^{16}$

As a final comment, we briefly compare the measured performance with those offered by three competing approaches: silicon nanowires, chalcogenide photoniccrystal waveguides, and quasi-phase-matched second-order nonlinear interactions in ferroelectric materials. By exploiting silicon nanowires, the reported values for $\gamma$ are generally between 100 and $350 \mathrm{~W}^{-1} \mathrm{~m}^{-1}$, ${ }^{17}$ which is lower than the here measured $\gamma_{\mathrm{TM}}$ by at least a factor 3 . On the other hand, considering materials not-compatible with CMOS technology, a very large value of $\gamma$ was found by operating near the band edge of a chalcogenide photonic crystal waveguide, ${ }^{9}$ but in that experiment, the conversion efficiency was drasti-

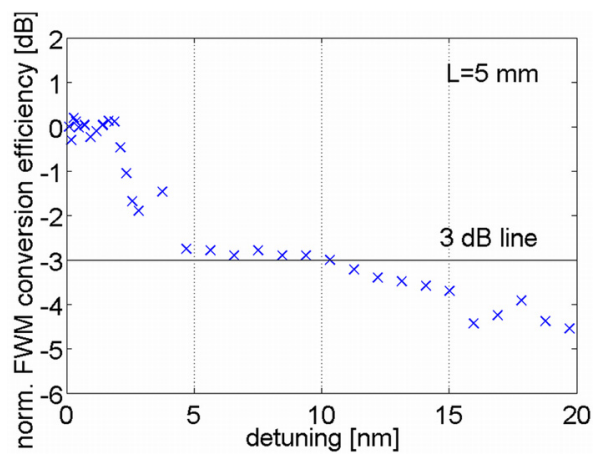

FIG. 4. (Color online) Conversion efficiency as a function of the pumpsignal detuning. cally dependent on pump wavelength. Moreover, the working bandwidth of a similar device is generally narrow, as the high- $\gamma$ effect is obtained only very close to the device bandedge, where the group index becomes very large. Finally, considering the performance of optical frequency converters based on quasi-phase-matched lithium-niobate (or lithiumtantalate) waveguides, it is important to highlight that this approach relies on a very mature technology and provides large conversion bandwidth, low waveguide losses, and high efficiency. On the other hand, these devices are not compatible with Si-based photonics, they have a very large foot-print and require a critical thermal stabilization of the chip. ${ }^{18,19}$

In conclusion, we reported a four-wave-mixing experiment, performed by using a low-loss Si:nc slot-waveguide, showing very large conversion efficiency. This result, combined with data obtained on the conversion bandwidth, indicates that this kind of structure is a promising candidate for the development of all optical processing devices.

University of Pavia authors would like to thank Professor Vittorio Degiorgio for his contribution to fruitful discussions and his useful comments. This work has been partially supported by the University of Pavia grant "Dottori di ricerca e mondo del lavoro."

${ }^{1}$ V. R. Almeida, Q. Xu, C. A. Barrios, and M. Lipson, Opt. Lett. 29, 1209 (2004).

${ }^{2}$ A. Di Falco, C. Conti, and G. Assanto, Opt. Lett. 31, 3146 (2006).

${ }^{3}$ J. C. G. De Sande, S. Stivala, J. Gonzalo, and G. Assanto, J. Nonlinear Opt. Phys. Mater. 15, 191 (2006).

${ }^{4}$ H. Ishikawa and T. Kondo, Appl. Phys. Express 2, 042202 (2009).

${ }^{5}$ A. S. Solntsev, A. A. Sukhorukov, D. N. Neshev, R. Iliew, R. Geiss, T. Pertsch, and Y. S. Kivshar, Appl. Phys. Lett. 98, 231110 (2011).

${ }^{6}$ C. Koos, P. Vorreau, T. Vallaitis, P. Dumon, W. Bogaerts, R. Baets, B. Esembeson, I. Biaggio, T. Michinobu, F. Diederich et al., Nature Photon. 3, 216 (2009).

${ }^{7}$ G. V. Prakash, M. Cazzanelli, Z. Gaburro, L. Pavesi, F. Icona, G. Franzò, and F. Priolo, J. Appl. Phys. 91, 4607 (2002).

${ }^{8}$ A. Martinez, J. Blasco, P. Sanchis, J. V. Galan, J. Garcia-Ruperez, E. Jordana, P. Gautier, Y. Lebour, S. Hernandez, R. Spano et al., Nano Lett. 10, 2288 (2010)

${ }^{9}$ J. Blasco, J. V. Galan, P. Sanchis, J. M. Martinez, A. Martinez, E. Jordana, J. M. Fedeli, and J. Marti, Opt. Commun. 283, 435 (2010).

${ }^{10}$ B. Kuyken, S. Clemmen, S. K. Selvaraja, W. Bogaerts, D. Van Thourhout, P. Emplit, S. Massar, G. Roelkens, and R. Baets, Opt. Lett. 36, 552 (2011).

${ }^{11}$ E. Jordana, J. M. Fedeli, P. Lyan, J. P. Colonna, P. E. Gautier, N. Daldosso, L. Pavesi, Y. Lebour, P. Pellegrino, B. Garrido et al., in Proceedings of the 4th IEEE International Conference Group IV Photonics, (Tokyo, Japan 2007).

${ }^{12}$ G. P. Agrawal, Nonlinear Fiber Optics, 2nd ed. (Academic Press, New York, 1995).

${ }^{13}$ V. Eckhouse, I. Cestier, G. Eisenstein, S. Combrié, P. Colman, A. De Rossi, M. Santagiustina, C. Someda, and G. Vadalà, Opt. Lett. 35, 1440 (2010).

${ }^{14}$ R. Spano, N. Daldosso, M. Cazzanelli, L. Ferraioli, L. Tartara, J. Yu, V. Degiorgio, E. Giordana, J. Fedeli, and L. Pavesi, Opt. Express 17, 3941 (2009).

${ }^{15}$ A. C. Turner, C. Manolatou, B. S. Schmidt, M. Lipson, M. A. Foster, J. E. Sharping, and A. L. Gaeta, Opt. Express 14, 4357 (2006).

${ }^{16}$ Y. Yue, L. Zhang, J. Wang, R. G. Beausoleil, and A. E. Willner, Opt. Express 18, 22061 (2010).

${ }^{17}$ T. Vallaitis, S. Bogatscher, L. Alloatti, P. Dumon, R. Baets, M. Scimeca, I. Biaggio, F. Diederich, C. Koos, W. Freude, and J. Leuthold, Opt. Express 17, 17357 (2009).

${ }^{18}$ P. Martelli, P. Boffi, M. Ferrario, L. Marazzi, P. Parolari, R. Siano, V. Pusino, P. Minzioni, I. Cristiani, C. Langrock et al., Opt. Express 17, 17758 (2009).

${ }^{19}$ G. Nava, P. Minzioni, I. Cristiani, A. C. Busacca, L. Curcio, S. Stivala, and G. Assanto, Electron. Lett. 46, 1686 (2010). 